\title{
IDENTIFICACIÓN DE GENES DE RESISTENCIA A CARBAPENÉMICOS EN ENTEROBACTERIAS DE HOSPITALES DE PERÚ, 2013-2017
}

\author{
Rosa Sacsaquispe-Contreras ${ }^{1, a}$, Henri Bailón-Calderón²,b
}

\begin{abstract}
RESUMEN
La diseminación global de carbapenemasas es de importancia en la salud pública. El objetivo del estudio es describir la presencia de genes de resistencia a carbapenémicos tipo KPC y metalobetalactamasas en enterobacterias aisladas de 12 hospitales y remitidos al Laboratorio de Referencia Nacional de Infecciones Intrahospitalarias del Instituto Nacional de Salud de Perú durante los años 2013 al 2017. Las cepas fueron identificadas por métodos convencionales, la resistencia antimicrobiana fue determinada por métodos fenotípicos, bioquímicos y la presencia de genes de resistencia se detectaron por PCR convencional. Se identificaron 83 cepas con carbapenemasas: $26(31,3 \%)$ portando el gen $b / a_{\mathrm{KPC}}, 56(67,5 \%)$ el gen $b l a_{\mathrm{NDM}}$ y una $(1,2 \%)$ cepa con el gen $b / a_{\text {IMP }}$. Es el primer reporte que da a conocer los genes de carbapenemasas circulantes en hospitales de Perú, por lo que se requiere mejorar la vigilancia para tener un mejor conocimiento de la situación en Perú.
\end{abstract}

Palabras Clave: Carbapenémicos; Infecciones por enterobacteriaceae; Resistencia betalactámica; Enterobacteriaceae Productoras de Carbapenemasa; Perú (fuente: DeCS BIREME)

\section{IDENTIFICATION OF CARBAPENEM-RESISTANT GENES IN ENTEROBACTERIA FROM PERUVIAN HOSPITALS, 2013-2017}

\begin{abstract}
The global spread of carbapenemases is a significant public health concern. The aim of this report is to describe the presence of KPC-type carbapenem-resistant genes and enterobacteria isolated in 12 hospitals and forwarded to the Peruvian National Institute of Health's National Infection Reference Laboratory during the period between 2013 and 2017. The strains were identified by conventional methods; antimicrobial resistance was determined by phenotypic and biochemical methods. The presence of resistant genes was detected by conventional PCR. Eighty-three (83) strains harboring carbapenemases were identified: $26(31.3 \%)$ carrying the $b l a_{\mathrm{KPC}}$ gene, $56(67.5 \%)$ the $b / a_{\mathrm{NDM}}$ gene, and one strain $(1.2 \%)$ with the $b a_{\text {IMP }}$ gene. This is the first report that shows the circulating carbapenemases genes in Hospitals in Peru of cases submitted for their confirmation to the National Reference Laboratory, so it is necessary to improve the surveillance to better understand their situation in our country.
\end{abstract}

Keywords: Carbapenems; Enterobacteriaceae infections; beta-Lactam Resistance; carbapenemase-producing Enterobacteriaceae; Peru (source: MeSH NLM)

\section{INTRODUCCIÓN}

Los carbapenémicos, entre los betalactámicos, son los más efectivos contra las bacterias grampositivas y gramnegativas, presentando un amplio espectro de actividad antibacteriana. Son altamente efectivos contra muchas especies bacterianas y menos vulnerables a la mayoría de los determinantes de la resistencia a betalactámicos. Además, son considerados como el último y más confiable recurso para tratar las infecciones bacterianas y son más seguros que otras drogas de última línea como las polimixinas; pues presentan pocos efectos adversos.

\footnotetext{
Laboratorio de Referencia Nacional de Infecciones Hospitalariamente. Centro Nacional de Salud Pública. Instituto Nacional de Salud. Lima, Perú. Laboratorio de Referencia Nacional de Biotecnología y Biología Molecular. Centro Nacional de Salud Pública. Instituto Nacional de Salud. Lima, Perú.

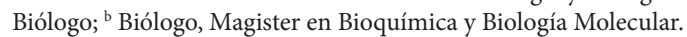

Recibido: 14/02/2018 Aprobado: 30/05/2018 En línea: 22/06/2018
} 
Por estas razones, la emergencia y rápida diseminación de las Enterobacterias Resistentes a Carbapenémicos (CRE) a nivel mundial constituye un problema de salud pública de gran importancia ${ }^{(1)}$. Las CRE también son identificadas como una fuente de infecciones adquiridas en la comunidad. Las enterobacterias productoras de carbapenemasas también son multidrogo resistentes explicando así la dificultad para su tratamiento y la falta de detección en portadores ${ }^{(2)}$.

Hasta hace poco tiempo, la resistencia a carbapenémicos era poco común en bacterias gramnegativas; sin embargo, en la actualidad el aumento de las bacterias resistentes a los carbapenémicos es considerado como uno de los problemas de salud pública más urgentes, debido a su uso frecuente para tratar las infecciones por bacterias gramnegativas $^{(3)}$

Las enterobacterias son causas comunes de infecciones asociadas a la atención hospitalaria incluyendo infecciones urinarias, infecciones del tracto sanguíneo en pacientes con catéteres, neumonía (frecuentemente asociadas a ventilación mecánica) ${ }^{(4,5)}$ aumentando así la posibilidad de propagación de CRE en la comunidad. Esto y las limitadas opciones terapéuticas disponibles para tratar a los pacientes infectados con estos organismos, han hecho que las CRE sean de importancia epidemiológica ${ }^{(3)}$. El aumento en el uso de carbapenémicos y probablemente con otros múltiples factores han contribuido al aumento dramático de CRE. Los pacientes internados por largo tiempo tienen un mayor riesgo de colonización e infección con CRE, aunque se requieren más estudios para evaluar si esto simplemente refleja una superposición de factores de riesgo, o si se produce una transmisión significativa de paciente a paciente ${ }^{(6)}$.

Los genes que codifican carbapenemasas, usualmente se encuentran en plásmidos o en otros elementos genéticos móviles. Estos, permiten al microorganismo adquirir resistencia a los antimicrobianos. Los resultados son aislamientos multiresistentes, extensivamente resistentes, o panresistentes ${ }^{(7)}$. La movilidad de estos elementos genéticos también ha permitido su dispersión en diversas enterobacterias tales como E. coli, Klebsiella oxytoca, Enterobacter, Serratia, y especies de Salmonella.

Las carbapenemasas descritas en las enterobacterias pertenecen a las cuatro clases de betalactamasas $(A, B, C$ y $D)$, según la clasificación de Ambler basadas en las secuencias moleculares. Las KPC son un grupo de carbapenemasas que pertenecen a la clasificación molecular de Bush 2f, las carbapenemasas como KPC son únicas entre las betalactamasas de clase $A$ porque contienen como centro activo una serinabetalactamasa ${ }^{\left({ }^{8}\right)}$. Las NDM son metalobetalactamasas que pertenecen a la clase B de Ambler, y al grupo 3 de la clasificación de Bush, estas son las carbapenemasas adquiridas de mayor importancia clínica por su capacidad de hidrolizar a todos los antibióticos

\section{MENSAJES CLAVE}

Motivación para realizar el estudio. La resistencia a carbapenémicos es un problema a nivel mundial, en el Perú no se tiene información sobre los genes implicados que causan esta resistencia, en qué microorganismos ocurre y cuál es su frecuencia.

Principales hallazgos. En los hospitales de Lima y provincias estudiados existen enterobacterias que portan los genes de resistencia $b l a_{\mathrm{KPC}}(31,3 \%), b l a_{\mathrm{NDM}}(67,5 \%)$, y $b l a_{\mathrm{IMP}}(1,2 \%)$.

Implicancias. La diseminación de cepas de enterobacterias con resistencia por carbapenemasas constituye una amenaza para la salud pública. Es importante contar con nuevas políticas en los sistemas de salud que permita un control oportuno para minimizar su impacto.

betalactámicos (excepto el aztreonam) y no ser inhibidas por los inhibidores de betalactamasas ${ }^{(8,9)}$.

El objetivo de este estudio fue describir la presencia de genes de resistencia a carbapenémicos tipo KPC y metalobetalactamasas en enterobacterias aisladas de hospitales de Perú. Esta información permitirá comprender mejor este tipo de resistencia y mejorar la vigilancia y control de las bacterias productoras de carbapenemasas en Perú.

\section{EL ESTUDIO}

\section{DISEÑO DE ESTUDIO Y POBLACIÓN}

Estudio descriptivo observacional de serie de casos realizado en el Laboratorio de Referencia Nacional (LRN) de Infecciones Intrahospitalarias del Instituto Nacional de Salud de Lima, Perú, donde se realizan las confirmaciones moleculares de resistencia a carbapenemasas. Se incluyeron cepas de enterobacterias resistentes a carbapenémicos (imipenem, meropenem o ertapenem) de procedimientos de rutina provenientes de doce hospitales de Lima, Trujillo, Arequipa y Junín pertenecientes a Ministerio de Salud (Hospitales generales e Institutos especializados), Seguro Social (EsSalud) y Fuerzas Armadas, desde el 2013 al 2017. Además, se incluyeron cepas provenientes de dos brotes de Proteus mirabilis y Klebsiella pneumoniae en dos hospitales de Lima.

\section{ESTUDIO MICROBIOLÓGICO}

Las cepas remitidas fueron sembradas en agar Tripticase soya y agar Mac Conkey para su aislamiento. La identificación bacteriana se realizó mediante la metodología del Laboratorio de Infecciones Intrahospitalarias del Instituto Nacional de Salud ${ }^{(9)}$. La susceptibilidad a los agentes antimicrobianos (imipenem y meropenem (Oxoid, USA)) se determinó por el método de disco difusión (11), la detección de la concentración inhibitoria mínima (CIM) por método de 
tira de gradiente (E-Test Biomerieux) y la interpretación según las recomendaciones del The Clinical \& Laboratory Standards Institute (CLSI) ${ }^{(12)}$.

\section{DETECCIÓN FENOTIPICA DE MECANISMOS DE RESISTENCIA}

El Test de Hodge modificado según las recomendaciones de la CLSI ${ }^{(12)}$ se realizó hasta el 2014. La detección bioquímica de la producción de carbapenemasa se realizó regularmente a partir del año 2015 y fue determinada por el método de blue carba ${ }^{(13)}$. Las pruebas fenotípicas para detección presuntiva de la presencia del mecanismo de resistencia se realizaron por el método de aproximación de discos (test de sinergia) entre Imipenem 10 ug (Oxoid) y meropenem 10 ug (Oxoid) con discos de ácido fenilborónico (Britania) y ácido etilendiaminotetraacético (EDTA (Britania)) el cual fue realizado siguiendo las recomendaciones del Instituto Carlos Malbran (comunicación oral).

\section{DETECCIÓNMOLECULARDEGENESDERESISTENCIA A CARBAPENÉMICOS}

La detección de genes bla ${ }_{\mathrm{KPC}}$ y de metalobetalactamasas $\left(b / a_{\mathrm{NDM}}, b l a_{\mathrm{VIM}}, b l a_{\mathrm{IMP}}\right)$ se realizó por Reacción en Cadena de la Polimerasa (PCR) convencional. Las reacciones de PCR fueron preparadas utilizando los cebadores (sintetizados por Invitrogen) descritos en la Tabla 1 , donde se señalan las secuencias de cada cebador y el tamaño del producto de PCR o amplicón. La concentración de los cebadores fue de $200 \mathrm{nM}$ en las reacciones tanto individuales como múltiplex, y se realizó siguiendo el protocolo del Instituto Carlos Malbran ${ }^{(14,15)}$. Los controles positivos utilizados para los genes bla $\mathrm{KPC}_{\mathrm{C}} \mathrm{y}$ de metalo betalactamasas fueron procedentes del Instituto Carlos Malbran y la cepa $\mathrm{E}$. coli ATCC 25922 fue utilizada como control negativo.

Para las metalobetalactamasas, a partir del 2017 los tres genes fueron analizados simultáneamente en PCR múltiplex. Los controles positivos utilizados para los genes $b / a_{\mathrm{KPC}}$ y de metalobetalactamasas $\left(b / a_{\mathrm{NDM}}, b / a_{\mathrm{VIM}}, b / a_{\mathrm{IMP}}\right)$

Tabla 1. Cebadores utilizados en este estudio

\begin{tabular}{llc}
\hline Gen & Secuencia $\left(5^{\prime}\right.$ - 3') & Amplicón \\
\hline \multirow{2}{*}{ KPC } & F: AAC AAG GAA TAT CGT TGA TG & \multirow{2}{*}{$916 \mathrm{pb}$} \\
& R: AGA TGA TTT TCA GAG CCT TA & \\
\multirow{2}{*}{ NDM } & F: AGC ACA CTT CCT ATC TCG AC & \multirow{2}{*}{$512 \mathrm{pb}$} \\
& R: GGC GTA GTG CTC AGT GTC & \\
& F: GGY GTT TWT GTT CAT ACW & \\
IMP & TCK TTY GA \\
& R: GGY ARC CAAACC ACT ASG & \multirow{2}{*}{$404 \mathrm{pb}$} \\
& TTA TCT \\
VIM & F: AGT GGT GAG TAT CCG ACA G & \\
& R: ATG AAA GTG CGT GGA GAC & \\
\hline
\end{tabular}

F: Cebador Forward, R: Cebador Reverso, KPC: Klebsiella pneumoniae carbapenemase, NDM: New Delhi metallo- $\beta$-lactamase, IMP: imipenemase metallo- $\beta$-lactamase, VIM: Verona integron-encoded metallo- $\beta$-lactamase fueron cepas procedentes del Instituto Carlos Malbran y la cepa E. coliATCC 25922 fue utilizada como control negativo.

\section{CONSIDERACIONES ÉTICAS}

Al tratarse de una investigación no realizada directamente en seres humanos, debido que se trabajaron con cepas procedentes de hospitales, no fue necesario la aprobación de un comité de ética institucional.

\section{RESULTADOS}

Las cepas que ingresaron al estudio provenían de diferentes muestras clínicas: orina (28 muestras), sangre (16 muestras), aspirado bronquial (cinco muestras), secreción de herida (cinco muestras), catéter venoso central (cinco muestras), secreción de dren (tres muestras), absceso (dos muestras), esputo (una muestra), secreción pancreática (una muestra), secreción de oído (una muestra), secreción bronquial (una muestra), tejido necrótico (una muestra), sin datos (14 muestras). Los hospitales que derivaron las cepas al LRN de Infecciones Intrahospitalarias se muestran en la Tabla 2.

En Lima se encontraron 55 cepas NMD, 24 cepas KPC y una cepa IMP. En Trujillo se detectó una cepa NMD, en Junín una cepa KPC y en Arequipa una cepa KPC.

Se confirmó la presencia de carbapenemasas en las siguientes enterobacterias: Escherichia coli, Klebsiella pneumoniae, Proteus mirabilis. Providencia rettgeri, Providencia stuartii, Enterobacter cloacae, Enterobacter aerogenes, Citrobacter freundii, los detalles por cada microorganismo se describen en la Tabla 3.

De las 83 cepas con carbapenemasas, 26 (31,3\%) fueron portadores del gen $b l a_{\mathrm{KPC}}, 56(67,5 \%)$ fueron portadores

Tabla 2. Procedencia de cepas de enterobacterias según año y tipo de gen identificado

\begin{tabular}{lccccc}
\hline Gen & $\mathbf{2 0 1 3}$ & $\mathbf{2 0 1 4}$ & $\mathbf{2 0 1 5}$ & $\mathbf{2 0 1 6}$ & $\mathbf{2 0 1 7}$ \\
\hline KPC & 1 & 6 & 2 & 5 & 12 \\
NDM & 1 & 10 & 5 & 14 & 26 \\
IMP & 0 & 0 & 0 & 0 & 1 \\
Total & 2 & 16 & 7 & 19 & 39 \\
\hline
\end{tabular}

2013: Hospital A. Loayza, Hospital Edgardo Rebagliati Martins 2014: Hospital Hipolito Unanue, Hospital Edgardo Rebagliati Martins 2015: Instituto Nacional de Enfermedades Neoplasicas, Hospital Hipolito Unanue, Hospital Cayetano Heredia, Hospital Edgardo Rebagliati Martins, Hospital A. Loayza, Hospital Regional de Trujillo

2016: Instituto Nacional de Enfermedades Neoplasicas , Hospital Dos de Mayo, Hospital Hipolito Unanue, Hospital Essalud de Huancayo 2017: Instituto Nacional de Enfermedades Neoplasicas, Hospital Dos de Mayo, Hospital Cayetano Heredia, Instituto Nacional de Rehabilitación, Hospital de la Fuerza Aerea del Perú, Hospital A. Loayza, Hospital Honorio Delgado de Arequipa

KPC: Klebsiella pneumoniae carbapenemase, NDM: New Delhi metallo$\beta$-lactamase, IMP: imipenemase metallo- $\beta$-lactamase 
Tabla 3. Distribución de genes de resistencia a carbapenémicos en cepas de enterobacterias

\begin{tabular}{|c|c|c|c|c|c|}
\hline \multirow{2}{*}{ Microorganismo } & \multicolumn{4}{|c|}{ Gen } & \multirow{2}{*}{ Total } \\
\hline & KPC & NDM & IMP & VIM & \\
\hline Escherichia coli & 2 & 1 & 0 & 0 & 3 \\
\hline Klebsiella pneumoniae & 14 & 40 & 1 & 0 & 55 \\
\hline Proteus mirabilis & 1 & 10 & 0 & 0 & 11 \\
\hline Providencia rettgeri & 0 & 4 & 0 & 0 & 4 \\
\hline Providencia stuartii & 0 & 1 & 0 & 0 & 1 \\
\hline Enterobacter cloacae & 7 & 0 & 0 & 0 & 7 \\
\hline Enterobacter aerogenes & 1 & 0 & 0 & 0 & 1 \\
\hline Citrobacter freundii & 1 & 0 & 0 & 0 & 1 \\
\hline
\end{tabular}

KPC: Klebsiella pneumoniae carbapenemase, NDM: New Delhi metallo- $\beta$-lactamase, IMP: imipenemase metallo- $\beta$-lactamase, VIM: Verona integronencoded metallo- $\beta$-lactamase

del gen bla ${ }_{\mathrm{NDM}}$ y uno $(1,2 \%)$ fue portador del gen $b / a_{\text {IMP }}$ en una cepa de Klebsiella pneumoniae aislada a partir de una muestra de sangre, (Figura 1). La mayor frecuencia de portadores del gen bla ${ }_{\mathrm{NDM}}$ podrían estar influenciados por un incremento de casos ocurrido en dos hospitales de Lima en 2016 y 2017.

Los genes bla ${ }_{\mathrm{KPC}}$ y $b / a_{\mathrm{NDM}}$ se detectaron en cepas procedentes de muestras de sangre, orina, esputo, absceso, secreción pancreática, tejido necrótico, secreción de oído, secreción de herida operatoria, dren de absceso, aspirado bronquial y catéter venoso central.

Específicamente, en cepas procedentes de fluidos corporales estériles, se encontró en orina nueve enterobacterias portadoras del gen $b l a_{\mathrm{KPC}}$ y 18 portadoras del gen $b a_{\mathrm{NDM}}$, mientras que en cepas procedentes de sangre se encontró seis enterobacterias portadoras del gen $b a_{\mathrm{KPC}}$, ocho con el gen blaNDM y una cepa con el gen gen bla ${ }_{\text {IMP }}$.

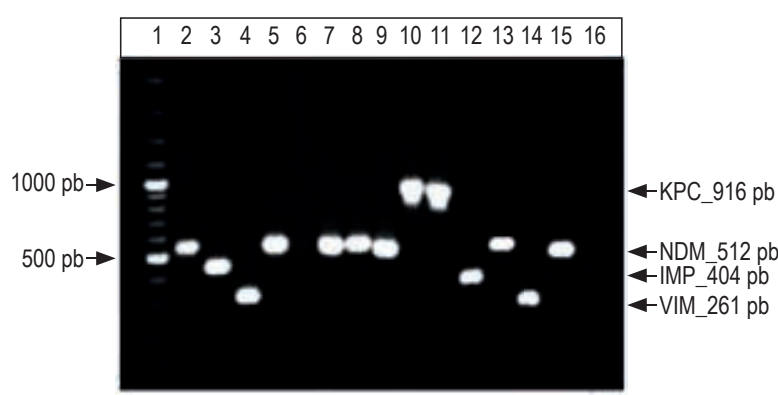

Figura 1. Detección de genes $b a_{\mathrm{KPC}}, b a_{\mathrm{NDM}}, b / a_{\mathrm{IMP}}, b l a_{\mathrm{VIM}}$ por PCR en algunas cepas. Corrida electroforética en gel de agarosa. Carriles: 1- Marcador de peso molecular (100pb), 2- Control

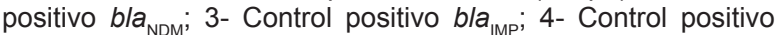
bla $_{\mathrm{VIM}}$; 5- IIH-46-17; 6- Control negativo; 7 - IIH-58 -17; 8- IIH73-17; 9- IIH-82-17 10- Control positivo bla $\mathrm{KPC}_{1}$ 11- IIH-70-17; 12- IIH-59-17; 13- IIH-93-17; 14- Control positivo bla ${ }_{\text {VIM }} 15-\mathrm{IIH}-$ 96-17; 16- Control negativo.

\section{DISCUSIÓN}

En 2006 en Colombia se notifica por primera vez la presencia de carbapenemasa tipo KPC ${ }^{(16)}$ y en 2011 en Guatemala se notifica la presencia de carbapenemasas NDM ${ }^{(17)}$. En Perú se han notificado casos de enterobacterias productoras de carbapenemasa principalmente por KPC ${ }^{(18)}$ y NDM (datos no publicados) desde el 2013. Asimismo, en 2016 se ha reportado la presencia de NDM en cepas de Klebsiella pneumoniae en un hospital de Lima ${ }^{(19)}$.

Durante el 2013 y 2014, las primeras confirmaciones fenotípicas de carbapenemasas en el LRN de Infecciones Intrahospitalarias se realizaron mediante el método de Hodge modificado; además, de la detección fenotípica mediante el test de sinergia de imipenem y meropenem con ácido fenilboronico y EDTA. El método de Hodge modificado tiene una baja especificidad y sensibilidad para la identificación de metalobetalactamasas tipo NDM, en comparación con otras carbapenemasas como las carbapenemasas tipo OXA o las serincarbapenemasas. Es por ello que a partir del 2015 se implementó el método bioquímico de blue carba para la determinación de carbapenemasas. Los resultados obtenidos con este método fueron positivos en todos los casos y la reacción fue positiva entre $1 \mathrm{~min}$ y $1 \mathrm{~h} 30 \mathrm{~min}$ resultando ser un método práctico, de fácil y rápida ejecución, recomendable para el uso en los laboratorios microbiológicos hospitalarios.

En 2014, un hospital reportó el incremento de cepas de Proteus mirabilis portadores del gen NDM, en 2016 otro hospital reportó el incremento de cepas de Klebsiella pneumoniae portadores del gen NDM, ambos reportes probablemente incrementaron los casos de enterobacterias portadoras del gen NDM $(67,5 \%)$ frente a KPC $(31,3 \%)$ y de IMP $(1,2 \%)$, esto es contrario a lo reportado por los sistemas de vigilancia de Colombia ${ }^{(20)}$, Chile ${ }^{(21)}$ y Canadá ${ }^{(22)}$, 
donde KPC es la carbapenemasa más frecuentemente detectada en estos microorganismos.

Después del descubrimiento de IMP en Japón en 1991, este fue detectado en muchas partes del mundo (en las enterobacterias resulta poco frecuente este gen), su presencia se ha confirmado en China, Japón, Taiwan, Australia, Filipinas, India, Tailandia, España y Brasil. Las especies más comúnmente asociadas a IMP en las enterobacterias incluyen Klebsiella pneumoniae, Escherichia coli, y Enterobacter spp. ${ }^{(23)}$. En este estudio se confirma por primera vez la presencia del gen $b a_{\text {IMP, }}$, en una cepa de Klebsiella pneumoniae.

Como limitación del estudio podemos mencionar la ausencia de información en algunas muestras remitidas, como la edad, el tipo de muestra, o el tipo de dispositivo, estos datos son importantes para mantener una adecuada vigilancia en este tipo de microorganismos. Asimismo, no se puede determinar el gen predominante que circula en Perú, por la falta de más información de otras regiones y porque se está reportando sólo los casos remitidos por los hospitales que solicitaron confirmación molecular.

Este estudio presenta la primera evidencia de genes de resistencia a carbapenémicos que circulan en nuestro medio, que se presentan como un problema de salud pública. Es necesario fortalecer las estrategias de vigilancia, prevención y control a nivel hospitalario, para evitar la diseminación de enterobacterias portadores de genes de resistentes a carbapenémicos, de lo contrario, en un futuro probablemente no contaremos con opciones terapéuticas viables, asumiendo el riesgo de incrementar la morbilidad y mortalidad de los pacientes.

Agradecimientos: Los autores agradecen al Instituto Carlos Malbran de Argentina por la verificación de las pruebas de PCR de los primeros casos, al personal de microbiología, infectología y epidemiología de los hospitales participantes, al personal profesional y técnico del LRN de Infecciones Intrahospitalarias quienes apoyaron en el procesamiento y control de calidad interno

Fuentes de financiamiento: Instituto Nacional de Salud de Perú

Conflictos de interés: ninguno

\section{REFERENCIAS BIBLIOGRÁFICAS}

1. Meletis G. Carbapenem resistance: Overview of the problem and future perspectives. Ther Adv Infect Dis. 2016;3(1):1521. doi: $10.1177 / 2049936115621709$.

2. Nordmann P, Carrer A. Les carbapénèmases des entérobactéries. Arch Pediatr. 2010;17 Suppl 4:S154-62. doi: 10.1016/ S0929-693X(10)70918-0.

3. Kelly A, Mathema B, Larson E. Carbapenem-resistant Enterobacteriaceae in the community: a scoping review. Int J Antimicrob Agents. 2017;50(2):127-134. doi: 10.1016/j.jijantimicag.2017.03.012.

4. Neuner E, Sekeres J, Hall G, Van Duin D. Experience with fosfomycin for treatment of urinary tract infections due to multidrugresistant organisms. Antimicrob Agents Chemother. 2012;56(11):5744-8. doi: 10.1128/AAC.00402-12.

5. van Duin D, Kaye KS, Neuner EA, Bonomo RA. Carbapenem-resistant Enterobacteriaceae: A review of treatment and outcomes. Diagn Microbiol Infect Dis. 2013;75(2):115-20. doi: 10.1016/j.diagmicrobio.2012.11.009.

6. Perez F, van Duin D. Carbapenemresistant Enterobacteriaceae: A menace to our most vulnerable patients. Cleve Clin J Med. 2013;80(4):225-33. doi: 10.3949/ ccjm.80a.12182.

7. Magiorakos AP, Srinivasan A, Carey RB, Carmeli Y, Falagas ME, Giske CG, et al. Multidrug-resistant, extensively drug-resistant and pandrug-resistant bacteria: an international expert proposal for interim standard definitions for acquired resistance. Clin Microbiol Infect. 2012;18(3):268-81. doi: 10.1111/j.14690691.2011.03570.x.

8. Bush K, Bush K, Jacoby G. Updated Functional Classification of $\beta$-Lactamases. Antimicrob Agents Chemother. 2010;54(3):969-76. doi: 10.1128/ AAC.01009-09.

9. Morrojon García M. Carbapenemasas, una amenaza actual. Rev Cub Med Int Emerg 2012;11(4) 2613-18.

10. Instituto Nacional de Salud. Manual de Procedimientos Bacteriológicos en Infecciones Intrahospitalarias. Serie de Normas Técnicas $\mathrm{N}^{\circ}$ 28. Lima: Ministerio de Salud, INS; 2001. Disponible en: ftp://ftp2.minsa.gob.pe/descargas/OGCI/proyectosterminados/Proyecto_vigia/Doc12.pdf

11. Instituto Nacional de Salud. Manual de Procedimientos para la Prueba de Sensibilidad Antimicrobiana por el Método de Disco Difusión. Serie de Normas Técnicas $\mathrm{N}^{\circ} 30$. Lima: Ministerio de Salud, INS; 2002. Disponible en: http://www.ins.gob.pe/insvirtual/ images/otrpubs/pdf/manual\%20 sensibilidad\%202.pdf

12. CLSI. Performance Standards for Antimicrobial Susceptibility Testing. 27th ed. CLSI supplement M100S. Wayne, PA: Clinical and Laboratory Standards Institute; 2017. Disponible en: http:// www.facm.ucl.ac.be/intranet/CLSI/ CLSI-2017-M100-S27.pdf

13. Instituto Nacional de Enfermedades Infecciosas, Administración Nacional de Laboratorios e Institutos de Salud. BLUE CARBA - Detección rápida de carbapenemasas directo de placas de cultivo [Internet]. Buenos Aires: INEI, ANLIS; 2014. [Citado el 1 de Febrero de 2018]. Disponible en: http://antimicrobianos.com. ar/ATB/wp-content/uploads/2014/10/ BLUE-CARBA-pdf

14. Protocolos [Internet]. Antimicrobianos. com.ar. Buenos Aires: Instituto Nacional de Enfermedades Infecciosas (INEI) - ANLIS «Dr. Carlos G. Malbran» [2 pantallas]. Disponible en: http:// antimicrobianos.com.ar/category/ protocolo/

15. Protocolos de detección de MBL multiplex [Internet]. Antimicrobianos. com.ar. Buenos Aires: Instituto Nacional de Enfermedades Infecciosas (INEI) - ANLIS «Dr. Carlos G. Malbran» [2 pantallas]. Disponible en: http:// antimicrobianos.com.ar $/ 2017 /$ ?cat $=42$

16. Villegas MV, Lolans K, Correa A, Suarez CJ, Lopez J, Vallejo M, et al. First Detection of the Plasmid-Mediated Class A Carbapenemase $\mathrm{KPC}-2$ in Clinical 
Isolates of Klebsiella pneumoniae from South America. Antimicrob Agents Chemother. 2006;50(8):2880-2. doi: 10.1128/AAC.00186-06. 17.

17. Pasteran F, Albornoz E, Faccone D, Gomez S, Valenzuela C, Morales M, et al. Emergence of NDM-1-producing Klebsiella pneumoniae in Guatemala. Journal of Antimicrobial Chemotherapy. 2012;67:1795-97.

18. Velásquez J, Hernández R, Pamo $\mathrm{O}$, Candiotti M, Pinedo Y, Sacsaquispe R, et al. Klebsiella pneumoniae resistente a los carbapenemes. Primer caso de carbapenemasa tipo KPC en Perú. Rev Soc Peru Med Interna. 2013;26(4):192-6.

19. Resurrección-Delgado C, MontenegroIdrogo JJ, Chiappe-Gonzalez A, VargasGonzales R, Cucho-Espinoza C, MamaniCondori DH, et al. Klebsiella pneumoniae
Nueva delhi metalo-betalactamasa en el Hospital Nacional Dos de Mayo. Lima, Perú. Rev Peru Med Exp Salud Pública. 2017;34(2):261-7. doi: 10.17843/ rpmesp.2017.342.2615.

20. Ovalle M, Sandra S, González M, Hidalgo M, Duarte C, Beltrán M. Resultados de la vigilancia nacional de la resistencia antimicrobiana de enterobacterias y bacilos Gram negativos no fermentadores en infecciones asociadas a la atención de salud, Colombia, 2012-2014. Biomedica. 2017;37(4):473485. doi: 10.7705/biomedica.v37i4.3432.

21. Instituto de Salud Pública de Chile. Boletín de Resistencia Antimicrobiana [Internet]. Santiago: Programa de control de infecciones asociadas a la atención en salud. Ministerio de salud; 2015 [Citado el 30 de enero de 2018]. Disponible en: http://www.ispch.cl/sites/default/files/ BoletinRam-30112015A_0.pdf
22. Lefebvre B, Lévesque $S$, Bourgault AM, Mulvey MR, Mataseje, Boyd D, et al. Carbapenem non-susceptible Enterobacteriaceae in Quebec, Canada: Results of Laboratory Surveillance Program (2010-2012). PLoS One. 2015 Apr 24;10(4):e0125076. doi: 10.1371/journal.pone.0125076.

23. Matsumura Y, Peirano G, Motyl MR, Adams MD, Chen L, Kreiswirth B et al. Global molecular epidemiology of IMPproducing Enterobacteriaceae. Antimicrob Agents Chemother. 2017;61(4). pii: e02729-16. doi: 10.1128/AAC.02729-16.

\footnotetext{
Correspondencia: Rosa Sacsaquispe Contreras Dirección: Cápac Yupanqui 1400, Jesús Maria, Lima 11, Perú

Teléfono: (511) 7481111, Anexo 2121

Correoelectrónico:rs_ins@yahoo.com,rsacsaquispe@ ins.gob.pe
}
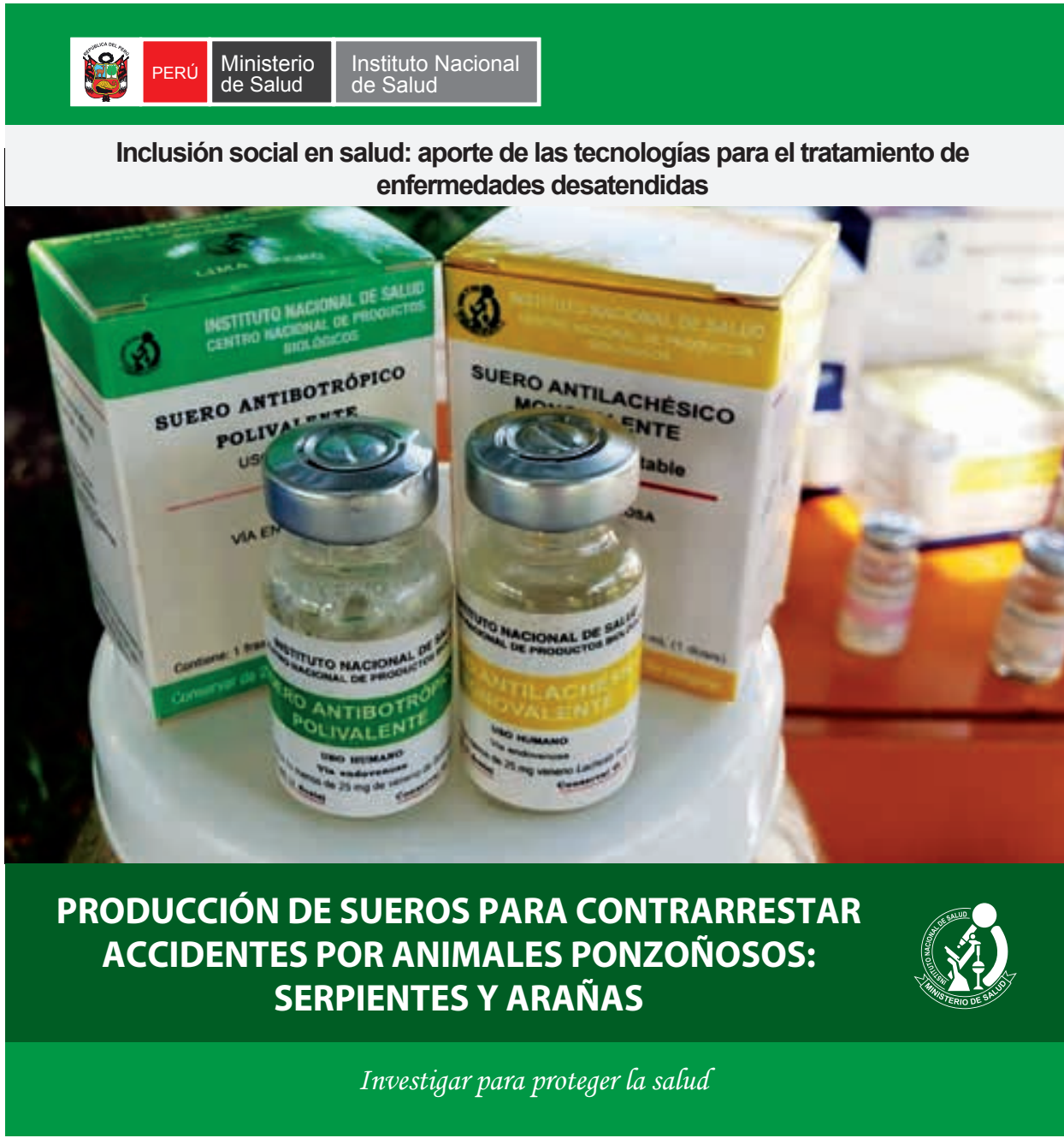